\title{
Can anterior approach be used for all patients with upper ureteric stones for shock wave lithotripsy?
}

\author{
Gopal Sharma ${ }^{1}$ iD
}

Received: 24 April 2020 / Accepted: 23 June 2020 / Published online: 27 June 2020

c) Springer-Verlag GmbH Germany, part of Springer Nature 2020

\section{Dear Editor,}

I read an article by Fathelbab et al. [1] with keep interest published in April 2020 edition of World Journal Urology. In this prospective randomized study, authors compared anterior versus posterior approach for shockwave lithotripsy (SWL) for upper ureteric stones located against L4 and L5 vertebrae. Authors noted anterior approach to be associated with significantly lower number of SWL sessions, lower pain scores, shorter time to stone expulsion, increased incidence of haematuria and infections. However, authors did not find any significant difference in terms of stone free rate and number of shocks. Authors have concluded that "anterior SWL to be the first choice for patients with upper ureter stones above iliac crests in mild to moderately obese patients". I would like to congratulate the authors for coming up with a technique for which the literature is lacking.

Bony prominences such as transverse process of lumbar vertebrae, ribs and iliac crests can indeed cause difficulty in targeting the stones in SWL. Alternative positions such as modified supine or prone have been tried in a bid to achieve better focusing of the stones especially in the upper ureter [2]. Posterior or classical approach is preferred for SWL as if offers direct path without intervening viscera due to retroperitoneal location of the kidney. Anterior approach described by authors has many limitations. First, focusing of stones can be difficult sometimes due to overlying bowel gases thus all the patients would require preprocedural bowel preparation which can incur extra costs. Second, focusing of such stones can be difficult if using is not impossible ultrasound and use of fluoroscopy can add on to the cumulative radiation doses

This comment refers to the article available online at https://doi. org/10.1007/s00345-020-03174-3.

Gopal Sharma

gopal.26669192@gmail.com

1 Department of Urology, Postgraduate Institute of Medical Education and Research, Chandigarh, India such patients receive. Third, abdominal fat pannus in obese patients can increase the skin to stone distance thus limiting the fragmentation even after achieving good focus. Lastly, even though rare there have been multiple reports of visceral injuries on following patients prone to SWL [3]. Furthermore, being a single centre study and limited to a specific patient population, firm conclusions cannot be reached. Because of such multiple limitations, I would like to differ from the conclusion that the authors have made. Until this approach is tested in a multicentric randomized study with adequate length of follow-up and power, anterior approach in supine position can be considered an alternative position for SWL in cases where stone focusing is difficult due to overlying bony prominences.

Acknowledgements None

Funding None to declare.

\section{Compliance with ethical standards}

Conflict of interest None to declare.

\section{References}

1. Fathelbab TK, Hasanein MGS, Fawzy AM (2020) Anterior or posterior SWL in proximal ureteral stones opposite to 4th and 5th lumbar vertebrae? World J Urol (Epub 2020/04/17)

2. Puppo P, Bottino P, Germinale F, Caviglia C, Ricciotti G (1988) Extracorporeal shock wave lithotripsy in the prone position for stones situated anteriorly. Eur Urol 15:113-117 (Epub 1988/01/01)

3. RodriguesNetto N Jr, Ikonomidis JA, Longo JA, RodriguesNetto M (2003) Small-bowel perforation after shockwave lithotripsy. J Endourol 17:719-720 (Epub 2003/12/04)

Publisher's Note Springer Nature remains neutral with regard to jurisdictional claims in published maps and institutional affiliations. 